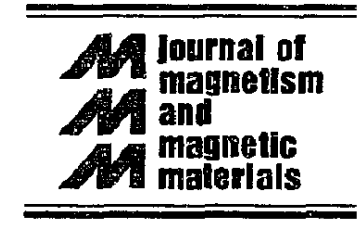

\title{
Stress measurements on magnetic multilayers
}

\author{
M.D. Bijker * , W.P. Van Drent, J.C. Lodder \\ MESA Research Institute, University of Twente, P.O. Box 217, 7500 AE, Enschede, The Netherlands
}

\begin{abstract} pressures and the stack composition of which has been clanged.

\section{Introduction}

Magnetic multilayers should in general exhibit a perpendicular magnetic anisotropy to be suitable for MO recording. One of the origins of this perpendicular magnetic anisotropy in thin films is caused by stress and magnetostriction. This effect seems to be especially pronounced in multilayered films like $\mathrm{Co}_{50} \mathrm{Ni}_{50} / \mathrm{Pt}$ multilayers [1]. Other authors have found considerable contributions of stress induced anisotropy in the order of $35 \%$ in e.g. $\mathrm{Co} / \mathrm{Pd}[2]$. Therefore it is interesting to further investigate the internal average filmstress of such multilayers.
\end{abstract}

We present stress measurements on $\mathrm{Co}_{50} \mathrm{Ni}_{50} / \mathrm{Pt}$ multilayers which have been sputtered at several argon and base

\section{Principle of stress measurement}

The internal average film stress can be measured by using the fact that due to the stress that is build in the thin film during deposition, the shape of the sample is changed. To measure this change in shape, a standard Michelson interferometer was used, extended with a phase sensitive method to enhance the accuracy of the system.

The difference in shape can then be characterised by the radii of curvature as depicted in Fig. 1. After Röll [3] the following formula is used to rclate the change in shape to the average film stress:

$$
\begin{aligned}
\left\langle\sigma_{i k}^{(2)}\right\rangle= & \frac{E_{1} H_{1}^{2}}{\sigma\left(1+m_{1}\right) H_{2}}\left[\frac{\partial^{2} W}{\partial x_{i} \partial x_{k}}\right. \\
& \left.+\frac{m_{1}}{1-m_{1}}\left(\frac{\partial^{2} W}{\partial x^{2}}+\frac{\partial^{2} W}{\partial y^{2}}\right) \delta_{i k}\right],
\end{aligned}
$$

with $\left\langle\sigma_{i k}^{(2)}\right\rangle$ in $\mathrm{Pa}$, the average internal film-stress tensor with $(i, k)=(x, y)$. To ensure isotropical elastic ptoperties of the substrate, we used silicon (111) [4] with Young

\footnotetext{
"Corresponding author. Fax: + 31-53-309547; e-mail: m.d.bij ker@el,utwente.nl.
}

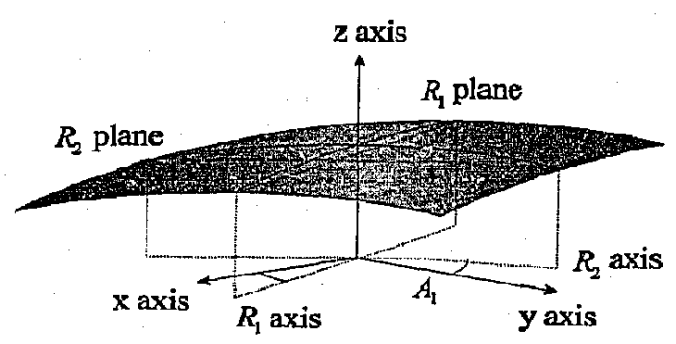

Fig. 1. Definition of the quantities $R_{1}, R_{2}$ and $A_{1}$ -

modulus $E_{1}=168.9 \mathrm{GPa}$, Poisson's constant $m_{1}=0.262$ and thickness $H_{1}=250 \mu \mathrm{m}$. Furthermore $H_{2}$ is the thickness of the film, $W(x, y)$ the difference in shape before and after deposition, $\partial^{2} W / \partial x^{2}=1 / R_{1}$ and $\partial^{2} W / \partial y^{2}=$ $1 / R_{2}$ the inverse radii of curvature in the $x$ and $y$ direction and $\delta_{i k}$ the Kronecker delta. Note that $\left\langle\sigma_{i k}^{(2)}\right\rangle$ is measured on the principle axis, i.e. $\left\langle\sigma_{x y}^{(2)}\right\rangle=0$. Note also that for $R_{1}=R_{2} E q$. (1) reduces 10 the well known equation given by Stoney [5].

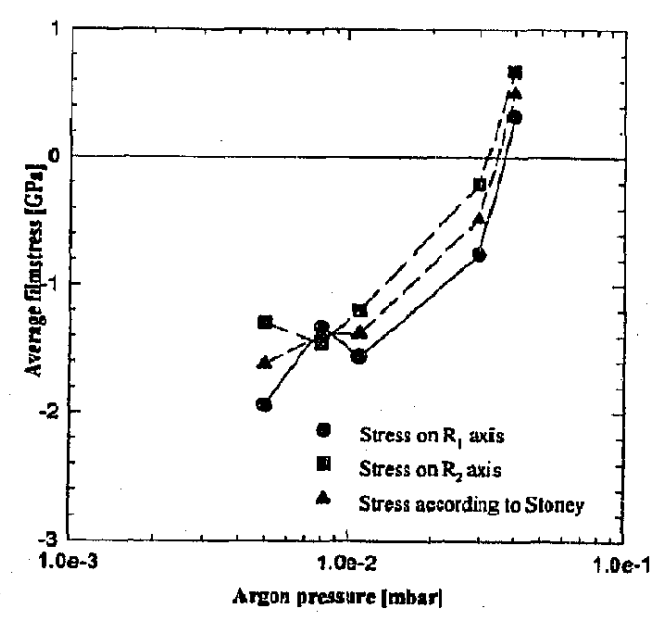

Fig. 2. Average internal film stress as a function of the argon pressure. 


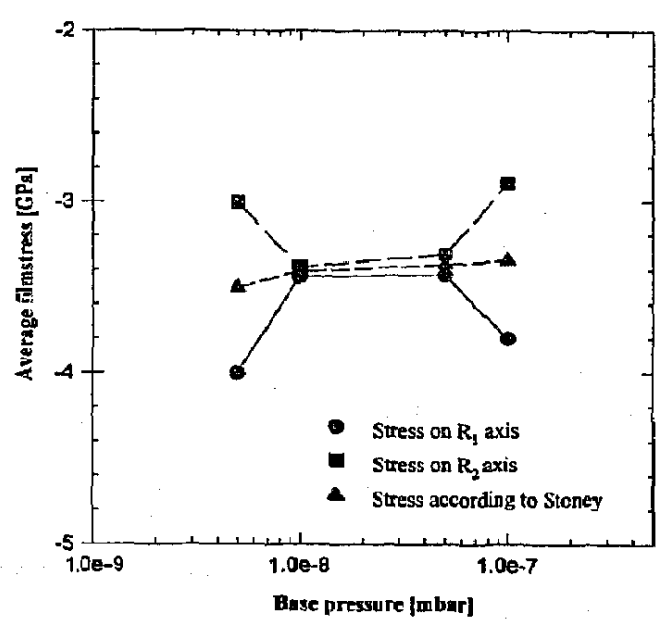

Fig. 3. Film stress as a function of the base pressure.

\section{Sample preparation}

All samples were deposited in an UHV sputtering system which makes use of sputter guns. The target to substrate distance was $10 \mathrm{~cm}$, the initial substrate temperature $27^{\circ} \mathrm{C}$ and the argon flow $35 \mathrm{ml} / \mathrm{min}$. The platinum was dc sputtered and the $\mathrm{Co}_{50} \mathrm{Ni}_{\text {so }}$ if sputtered both at 50 W. Furthermore all the individual layer thicknesses are shutter controlled. All multilayer films consist of a seedlayer of platinum, followed by $n \mathrm{Co}_{50} \mathrm{Ni}_{50} / \mathrm{Pt}$ bilayers.

To investigate the average film stress we prepared three film series, as listed in Table 1. With these series we investigated the influence of sputter-gas pressure, background pressure and stack composition on the average internal film stress.

Film thickness measurements were performed by measuring the deposition rate on single layers with DEKTAK and Low Angle XRD (LAXRD). The bilayer thickness of the deposited samples was also measured with LAXRD. From this the individual layer thicknesses were calculated as shown in the table.

\section{Results, díscussion and conclusion}

Before the measurements are presented, it should be mentioned that the total error in the measured radii of

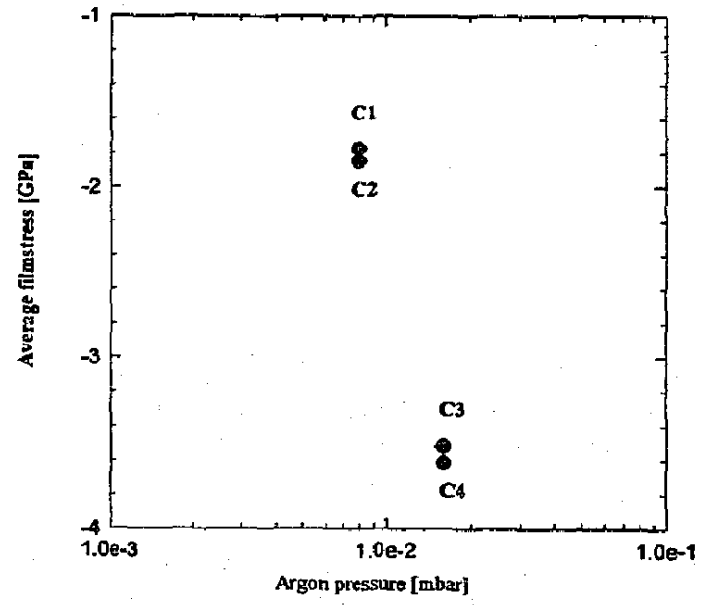

Fig. 4. Film stress according to Stoney as a function of the number of interfaces. The data points correspond with the table.

curvature is about $10 \%$. This results in an error in the internal average film stress of around $20 \%$.

In Fig. 2 the internal average film stress is plotted as a function of the applied argon pressure (series $A$ ). The stress changes sign (from compressive to tensile) at about $3.7 \times 10^{-2}$ mbar. The shape of the curve is in accordance with other results reported in literature [e.g. 6], however a physical explanation is still hardly known. Also some High Angle XRD measurements were performed on these samples. This data was found to be in agreement with the interferometrical results as presented here; the position of the multilayer peak follows the same trend from compressive to tensile.

In series B (see Fig. 3), film stress was studied as a function of the base pressure. From this we hardly see any dependence on the base pressure in the range from $5 \times$ $10^{-9}$ to $1 \times 10^{-7}$ mbar.

In series $\mathbf{C}$ the dependence of the composition of the stack was investigated. Here two films were sputtered at the same argon pressure but with twice the number of bilayers. Each time the total and the seed-layer thicknesses were kept constant. This experiment was done at two different argon pressures, as listed in Table 1. Note that the absolute value of the film stress is different since the total

Table 1

Different deposition parameters of film series A, B and C

\begin{tabular}{|c|c|c|c|c|c|c|}
\hline Series & $P_{A,}[\mathrm{mbar}]$ & $P_{\text {base }}$ [mbar] & $n$ & $t_{\text {seed }}[\AA ̊ 0]$ & $t_{\mathrm{CoN \textrm {N }}}[\mathbf{\mathrm { A }}]$ & $t_{\mathrm{Pt}}[\AA]$ \\
\hline$\overline{\mathbf{A}}$ & $5 \times 10^{-3}-4 \times 10^{-2}$ & $<5 \times 10^{-8}$ & 17 & 235 & 6.5 & 14.7 \\
\hline $\mathbf{B}$ & $1.6 \times 10^{-2}$ & $5 \times 10^{-9}-1 \times 10^{-7}$ & 17 & 235 & 6.5 & 14.7 \\
\hline $\mathrm{C} \# 1$ & $8 \times 10^{-3}$ & $<5 \times 10^{-8}$ & 17 & 180 & 13 & 10 \\
\hline$\# 3$ & $1.6 \times 10^{-2}$ & $<5 \times 10^{-8}$ & 17 & 235 & 13 & 29.4 \\
\hline$\# 4$ & $1.6 \times 10^{-2}$ & $<5 \times 10^{-8}$ & 34 & 235 & 6.5 & 14.7 \\
\hline
\end{tabular}


thickness with each argon pressure is different. The results are plotted in Fig. 4 and the striking fact is that the film stress has hardly any dependence on the chosen multilayer stack, i.e. the number of interfaces.

In conclusion we can say that the internal average film stress of the multilayers reported upon here show an expected behaviour as a function cf the argon pressure but seems hardly dependent on either the base pressure or the number of interfaces of the multilayer stack.

\section{References}

[1] W.P. Van Drent, Q. Meng, J.C. Lodder, U. Enz, Th.J.A. Popma, 14th ICMFS/ $\varepsilon$-MRS (Poster A 118).

[2] S. Hashimoto, Y. Ochiai, K. Aso, J. Appl. Phys. 66 (1989) 4909.

[3] K. Röll, J. Appl. Phys. 47 (1976) 3224.

[4] W.A. Brantley, J. Appl. Phys. 44 (1973) 534.

[5] G.G. Stoney, Proc. Roy. Soc. A32 (1909) 172.

[6] T. Yoshihara, K. Suzuki, J. Vac. Sci. Techn. B 11 (1993) 301. 